\title{
Cadre de travail national sur la santé pulmonaire : occasion d'analyse comparative entre les sexes
}

\author{
N Hemsing, M.A. (1); L Greaves, Ph.D. (2)
}

\section{Résumé}

Les maladies respiratoires liées au tabagisme représentent un énorme fardeau social et économique pour les femmes comme pour les hommes au Canada. Le présent article porte sur la contribution potentielle du Cadre de travail national sur la santé pulmonaire à la réduction des inégalités en santé respiratoire entre les femmes et les hommes et entre les sous-populations de femmes et d'hommes. Une analyse préliminaire des documents-cadres existants indique que les facteurs, les différences et les influences liés aux sexes n’ont pas encore été clairement ou suffisamment élucidés. Néanmoins, il y a des questions liées aux sexes en matière de prévention du tabagisme et d'abandon du tabac, de santé et de maladies pulmonaires. En particulier, nous traitons des besoins et des expériences des femmes en matière de santé respiratoire pour montrer le besoin d'une analyse comparative entre les sexes à l'intérieur du cadre. Par exemple, bien que les données probantes concernant les taux d'abandon ne soient pas concluantes, les femmes et les hommes n'ont ni les mêmes tendances d'abandon ni les mêmes raisons de fumer. La création d'une approche à la santé pulmonaire propre au Canada est une initiative importante, mais il faut s'occuper expressément des questions liées aux sexes en matière de maladies et de santé respiratoires aux étapes de la planification et de l'élaboration du cadre afin qu'il ait un effet bénéfique et durable sur les femmes et les hommes.

\section{Mots clés : tabagisme, maladies respiratoires, Cadre de travail national sur la santé pulmonaire (CTNSP), sexes, femmes}

\section{Introduction}

Les maladies respiratoires représentent un énorme fardeau social et économique au Canada. Le cancer du poumon, la bronchopneumopathie chronique obstructive (BPCO) et la pneumonie, les trois maladies respiratoires principales reliées aux décès au Canada, étaient la cause de 15,6\% des décès chez les hommes et de 13,5\% des décès chez les femmes en $2004^{1}$. La planification et l'élaboration actuelles d'un cadre de travail national sur la santé pulmonaire (CTNSP) constituent une étape essentielle de l'amélioration de la santé respiratoire des Canadiens. Ce cadre a été le fruit d'un atelier intitulé " Défi Pulmon-Air », qui s'est tenu en mars 2006 et qui a réuni les intervenants dans le but d'élaborer un plan d'action national pour améliorer la santé respiratoire au Canada. Sous les auspices de l'Association pulmonaire canadienne, le processus est dirigé par un comité directeur provisoire qui a coordonné les ateliers subséquents afin de guider le processus d'élaboration du cadre ${ }^{2}$. En août 2008, le document sur le Cadre de travail national sur la santé pulmonaire a été publié. Il servira à créer un plan d'action et à guider les décideurs et les intervenants lors de la planification stratégique ${ }^{3}$.

La création d'un plan d'ensemble pourrait améliorer la santé respiratoire des femmes et des hommes au Canada, de la prévention au diagnostic, à la gestion de la maladie et au traitement. Le cadre doit également servir à relever certains des défis en matière de santé auxquels sont confrontées diverses sous-populations de Canadiens. Par exemple, les documents produits au cours du processus d'élaboration du cadre expriment la nécessité de " [s'occuper] des besoins des populations vulnérables » et on y mentionne souvent les membres des Premières nations, les jeunes et les immigrants comme étant des sous-populations importantes pour les initiatives en matière de santé respiratoire ${ }^{4}$. Les quatre stratégies clés du document-cadre ${ }^{\mathrm{i}}$, qui portent tant sur la promotion de la santé que sur le dépistage des maladies, les politiques et la recherche, indiquent que les actions doivent avoir pour but non seulement d'améliorer la santé dans son ensemble, mais aussi de réduire les inégalités entre les populations autochtones et non autochtones $^{3}$. Le comité directeur a relevé de nombreuses questions fondées sur la recherche et sur la pratique, comme l'importance d'étudier les relations entre la santé respiratoire, les populations vulnérables et les facteurs environnementaux, et le besoin d'améliorer le soutien aux fournisseurs

i 1) Promotion de la santé, sensibilisation et prévention des maladies, 2) Dépistage et gestion des maladies, 3) Politiques, partenariats et soutien aux communautés et aux systèmes, 4) Recherche, surveillance et application des connaissances. 
de soins et aux patients et d'accroître l'usage de la spirométrie comme outil de diagnostic ${ }^{3,4}$.

L'élaboration d'un cadre national est un défi important étant donné la vaste gamme de maladies respiratoires aiguës et chroniques qui touchent les Canadiens. Celles-ci comprennent des maladies aussi diverses que l'asthme, la tuberculose, l'apnée du sommeil, la pneumonie, la grippe, la bronchopneumopathie chronique obstructive (BPCO) et le cancer du poumon; et la cause, le diagnostic, la gestion de la maladie et le traitement sont différents pour chacune. De plus, la cause, le diagnostic, la gestion de la maladie et le traitement ne sont pas les mêmes pour les femmes que pour les hommes et varient d'une sous-population de femmes ou d'hommes à l'autre. Ainsi, les questions liées aux sexes associées aux maladies et à la santé respiratoires doivent être traitées expressément dans le cadre afin qu'il ait un effet bénéfique et équitable tant sur les femmes que sur les hommes. Cependant, une analyse préliminaire des documents-cadres ${ }^{2-5}$ existants indique que les facteurs relatifs aux sexes, et les différences et les influences liées à la diversité n’ont pas été suffisamment cernées. Il est évident que l'analyse comparative entre les sexes n’y a pas été relevée comme étant un outil analytique essentiel pour la planification stratégique. En réponse à cette lacune, le présent article indique certains des besoins des femmes en santé respiratoire pour montrer comment ces omissions dans le cadre actuel peuvent empêcher de déceler les différences liées aux sexes.

\section{But de l'intégration de l'optique des sexes}

Pour que des améliorations importantes soient apportées à la santé pulmonaire, l'analyse comparative entre les sexes doit devenir une partie intégrante des initiatives en matière de planification et d'élaboration de programmes. Lanalyse comparative entre les sexes constitue un outil qui favorise l'examen d'une gamme de questions relatives au processus de recherche et à l'application des connaissances dans les activités d'élaboration de programmes ou de politiques comme le CTNSP. L'analyse comparative entre les sexes est recommandée dans la Stratégie pour la santé des femmes de Santé Canada ${ }^{6}$ et est également intégrée dans le travail de l'Organisation mondiale de la Santé (OMS) ${ }^{7}$. L'utilisation d'une telle approche nous aide à mieux comprendre comment les influences des sexes (aspects biologique, social et culturel) déterminent la santé et la maladie. L'efficacité de notre conception et de notre mise en œuvre de politiques et de programmes qui tiennent compte de la spécificité des sexes est déterminée en partie par de telles analyses ${ }^{8}$. L'utilisation de l'analyse comparative entre les sexes nous permettrait de nous pencher, dans le cadre de la stratégie nationale, sur la distribution inégale des maladies chez les femmes et les hommes et chez les sous-populations de femmes et d'hommes, y compris chez les Autochtones et les personnes à faible revenu.

Les documents examinés qui sous-tendent le CTNSP $\mathrm{P}^{2,4,5}$ n'expriment pas clairement ou de façon cohérente une approche fondée sur les sexes. Ils n'indiquent pas non plus si l'on a tenu compte des sexes dans les processus d'élaboration ou de mise en œuvre. À l'occasion, les différences entre les besoins et les problèmes des femmes et des hommes en matière de santé respiratoire sont traitées. De plus, l'accroissement du taux du tabagisme chez les femmes est relevé comme étant une question d'actualité ${ }^{3,5}$, tout comme la prévalence croissante chez les femmes de la bronchopneumopathie chronique obstructive et du cancer du poumon, en partie en raison du retard relatif des femmes sur les hommes en matière de tabagisme ${ }^{3}$. De plus, les femmes enceintes sont mentionnées comme étant une population importante en ce qui concerne la création des programmes d'abandon ${ }^{5}$. Toutefois, les femmes ne sont mentionnées dans les documents du CTNSP que de façon accessoire et sporadique par rapport aux autres populations, comme les jeunes, les membres des Premières nations et les immigrants $^{3,4}$. La nécessité d'examiner les besoins particuliers en matière de santé des femmes et des hommes et des souspopulations de femmes et d'hommes n'est généralement pas relevée. Le fait que toutes les populations sont divisées en sexes et que leurs problèmes de santé doivent être traités en conséquence a été omis dans le CTNSP.

\section{Influences des sexes sur la santé pulmonaire}

Il y a de nombreux facteurs et influences liés aux sexes dont il faut tenir compte pour élaborer un cadre qui correspond aux besoins en santé respiratoire. Pour illustrer ce point, nous examinons certains de ces besoins en mettant l'accent sur le contexte des problèmes de prévention, de diagnostic et de traitement des maladies respiratoires chez la femme. Par exemple, de nombreuses maladies respiratoires ne touchent pas les femmes et les hommes dans la même proportion. Le taux d'asthme est plus élevé chez les femmes que chez les hommes, la bronchopneumopathie chronique obstructive devient de plus en plus une maladie qui touche les femmes et les taux de mortalité à la suite du cancer du poumon augmentent chez les femmes depuis 1987 au Canada alors qu'ils diminuent chez les hommes ${ }^{1}$. Il y a aussi plus de cancers du poumon chez les femmes qui n’ont jamais fumé que chez les hommes qui n'ont jamais fumé9,10. De plus, certaines sous-populations de filles et de femmes, y compris les groupes de statut socio-économique inférieur et les minorités qui ne sont pas de race blanche ont des taux disproportionnellement élevés de maladies respiratoires. Les femmes qui ne sont pas de race blanche et ayant un faible revenu ont généralement un accès plus limité aux ressources en santé et souffrent plus souvent des maladies et des handicaps que les autres groupes ${ }^{11,12}$.

L’usage du tabac est un facteur clé dans le développement des maladies respiratoires. Les tendances des sexes en matière de tabagisme et d'exposition à la fumée et les facteurs biologiques, hormonaux et génétiques se chevauchent et influencent la susceptibilité des femmes aux maladies respiratoires $^{13}$. Les œstrogènes peuvent influencer la métabolisation de la fumée de cigarette et accroître ainsi ses effets néfastes ${ }^{10,12,14-16}$. Les données probantes montrent que les femmes qui fument moins que les hommes présentent des niveaux semblables de réduction de la fonction pulmonaire et que le tabagisme réduit davantage la fonction pulmonaire chez les femmes que chez les hommes ${ }^{12,17,18}$. Entre-temps, le marketing du tabac a été 
effectué en fonction des sexes et a ciblé efficacement et de façon croissante les femmes en montrant le tabagisme comme une activité prestigieuse qui permet de demeurer mince ${ }^{19,20}$. L'industrie du tabac a également mis au point des produits du tabac propres à chaque sexe. On a vendu à beaucoup de femmes des cigarettes "légères " contenant de plus fortes doses de nitrosamines que les autres et qui pourraient être l'une des causes de l'accroissement du taux de cancer du poumon chez les femmes $^{12}$. La fumée secondaire n'a pas non plus le même effet sur les femmes que sur les hommes, car, en raison du retard des femmes sur les hommes dans les tendances générales du tabagisme, il y a davantage de femmes non fumeuses qui vivent avec des hommes qui fument ${ }^{19,21}$.

Il y a aussi des questions liées aux sexes associées au diagnostic. Les femmes et les hommes ne signalent pas les mêmes symptômes. Celles-ci contractent la bronchopneumopathie chronique obstructive plus tôt que les hommes ${ }^{12}$. Elles sont également moins susceptibles que les hommes de signaler la production d'expectorations en raison des normes et idéaux liés aux sexes ${ }^{22}$. De même, certaines maladies sont souvent mal ou sous-diagnostiquées chez les femmes en raison de ces différences de présentation observées ainsi que d'un préjugé lié aux sexes dans le système de santé. Par exemple, l'asthme est plus souvent diagnostiqué chez les femmes atteintes de bronchopneumopathie chronique obstructive que chez les hommes ${ }^{13,23}$. De plus, même lorsque les femmes et les hommes présentent les mêmes symptômes, les fournisseurs de soins peuvent ne pas interpréter ces symptômes de la même manière ${ }^{24}$.

En général, les femmes qui ont des maladies respiratoires signalent plus d'hospitalisations, plus de limitations de leurs activités et de plus hauts taux d'anxiété et de dépression associés aux maladies respiratoires que les hommes ${ }^{1,23,25-29}$. Les changements d'apparence physique associés à la bronchopneumopathie chronique obstructive et au cancer du poumon peuvent être particulièrement troublants pour les femmes qui sont encouragées à respecter des normes sociales de beauté qui sont fonction du $\operatorname{sexe}^{28}$. Enfin, il a été montré que la réadaptation pulmonaire était plus efficace pour les hommes que pour les femmes au fil du temps ${ }^{22}$. Tous ces facteurs influencent la santé pulmonaire des femmes et des hommes, de la prévention au traitement, et il faut en tenir compte et les inclure lors de l'élaboration d'un cadre qui servira à améliorer sensiblement la santé respiratoire des femmes et des hommes.

\section{Conclusion}

Le CTNSP peut améliorer sensiblement la transmission et l'adoption des connaissances liées à la santé respiratoire en établissant des liens entre la recherche propre aux sexes sur l'usage du tabac et l'exposition à celuici et la santé et les maladies respiratoires. Il y a un besoin manifeste d'intégration des sphères de connaissances sur le tabac, les sexes et les maladies respiratoires ${ }^{12}$. Les taux de mortalité les plus élevés sont liés à des maladies associées au tabagisme ou à l'exposition à la fumée, comme la bronchopneumopathie chronique obstructive et le cancer du poumon. Les données concernant l'existence de différences entre les sexes en ce qui a trait à l'abandon du tabagisme ne sont pas concluantes ${ }^{\mathrm{ii}}$. Néanmoins, les femmes et les hommes ne fument pas pour les mêmes raisons et ont des tendances d'abandon différentes ${ }^{20}$. La création d'un groupe de travail du CTNSP sur les questions liées aux sexes et à la diversité, qui comprendrait des chercheurs et des décideurs des domaines de la santé pulmonaire, de la santé des femmes, de la santé des hommes, de l'usage du tabac et de la prévention du tabagisme, est une stratégie possible pour traiter certaines de ces questions. Les liens doivent être renforcés entre la recherche, les programmes et les politiques afin que les conclusions propres aux sexes qui se dégageront puissent être appliquées efficacement dans les cadres des soins de santé et par les décideurs ${ }^{40}$. En traitant ces facteurs et d'autres liés aux sexes, le CTNSP pourrait ouvrir la voie à un soutien efficace à tous les groupes « vulnérables ».

Le fait de relever l'analyse comparative entre les sexes comme étant un outil analytique clé encouragerait les chercheurs, les décideurs et les autres intervenants à tenir compte de ces différences. En résumé, les raisons pour lesquelles les femmes fument ainsi que les diverses expériences de soins et de traitement des maladies respiratoires diffèrent de celles des hommes et varient d'une sous-population de femmes à l'autre. Il a été question de certains des problèmes de santé respiratoire propres aux femmes qui montrent la nécessité d'une perspective détaillée, cohérente et généralisée des différences entre les sexes pour guider le CTNSP et le plan d'action. La mise en œuvre complète et détaillée d'une analyse comparative entre les sexes nécessiterait aussi une étude des besoins particuliers des hommes en matière de santé respiratoire et faciliterait une évaluation systématique des réactions fondées sur le sexe qui ciblent les hommes. Le CTNSP peut chercher à améliorer la santé respiratoire de tous les groupes par la création d'initiatives visant à soutenir et à encourager la réalisation d'autres recherches et interventions fondées sur les sexes. Bien que très peu de recherches aient porté sur les problèmes de santé respiratoire particuliers des souspopulations, comme les personnes à faible revenu et les membres de certains groupes ethniques, le CTNSP peut permettre de traiter ces questions et d'y réagir en utilisant une analyse comparative entre les sexes pour encourager la réflexion sur les femmes au Canada et orienter le Canada vers une recherche, des programmes, des politiques et des analyses futurs fondés sur les sexes.

ii Bien que les données de l'Enquête de surveillance du tabac au Canada (2006) montrent qu'au Canada, les femmes ont des taux d'abandon légèrement plus élevés $(60,1 \%)$ que les hommes $(57,9 \%)$, les études sur les différences de taux d'abandon entre les sexes révèlent souvent que les femmes ont des taux d'abandon plus faibles, plus de mal à cesser de fumer que les hommes et des taux de rechute plus élevés ${ }^{30-36}$. Toutefois, d'autres données suggèrent que les différences entre les sexes sont minimes ou inexistantes ${ }^{37,38}$ ou que les hommes ont en fait des taux d'abandon plus faibles et plus de mal à cesser de fumer que les femmes ${ }^{39}$. 


\section{Remerciements}

Le présent commentaire repose sur un examen des données probantes sur la santé respiratoire des femmes préparé pour le Population and Public Health Program de la Provincial Health Services Authority (PHSA). Les activités du Centre d'excellence pour la santé des femmes de la Colombie-Britannique sont rendues possibles par une contribution financière de Santé Canada. Cependant, les opinions exprimées dans le présent document sont celles des auteures et pas nécessairement celles de la PHSA ou de Santé Canada.

\section{Références}

1. Public Health Agency of Canada. Life and breath: respiratory disease in Canada, Ottawa, Public Health Agency of Canada, 2007.

2. Canadian Lung Association. Leading. Acting. Together. Building Canada's first national lung health action plan: report from the all-stakeholder workshop on lung health, Ottawa, Canadian Lung Association, 2007.

3. Canadian Lung Association. National lung health framework, Ottawa, Canadian Lung Association, 2008.

4. Canadian Lung Association. Respiratory health in Canada asset map: preliminary report, Ottawa, Canadian Lung Association, 2007.

5. de Lusignan S, Chan T, Cohen A, Thana L, Hague N, van Vlymen J. Health education and prevention for people with severe mental illness: a cross-sectional study of general practice computer records. Primary care mental health. 2005;3(3):221-33.

6. Health Canada. Women's health strategy, Ottawa, Health Canada,1999.

7. World Health Organization. TheWHOgender mainstreaming strategy, Geneva, World Health Association, 2008. URL : http:// www.who.int/gender/mainstreaming/ strategy/en/index.html.
8. Health Canada. Exploring concepts of gender and health, Ottawa, Health Canada, 2003.

9. Patel JD, Bach PB, Kris MG. Lung cancer in US women: a contemporary epidemic. JAMA. 2004;291:1763-768.

10. Tang DL, Rundle A, Warburton D, et al. Associations between both genetic and environmental biomarkers and lung cancer: evidence of a greater risk of lung cancer in women smokers. Carcinogenesis. 1998;19: 1949-953.

11. Steele CB, Richmond-Reese V, Lomax S. Racial and ethnic disparities in HIV/AIDS, sexually transmitted diseases, and tuberculosis among women. J Womens Health. 2006;15(2):116-22.

12. Greaves LJ, Richardson LA. Tobacco use, women, gender, and chronic obstructive pulmonary disease: are the connections being adequately made? Proc Am Thorac Soc. 2007; 4:675-79.

13. Caracta C. Gender differences in pulmonary disease. Mt Sinai J Med. 2003; 70(4):215-24.

14. Zang EA, Wynder EL. Differences in lung cancer risk between men and women: examination of the evidence. J Natl Cancer Inst. 1996;88(3/4):183-92.

15. Ben-Zaken Cohen S, Pare PD, Man SFP, Sin DD. The growing burden of chronic obstructive pulmonary disease and lung cancer in women: examining sex differences in cigarette smoke metabolism. Am J Respir Crit Care Med. 2007;176:113-20.

16. Sin DD, Cohen SB-Z, Day A, Coxson H, Pare PD. Understanding the biological differences in susceptibility to chronic obstructive pulmonary disease between men and women. Proc Am Thorac Soc. 2007;4:671-74.

17. Prescott E, Bjerg AM, Andersen PK, Lange P, Vestbo J. Gender difference in smoking effects on lung function and risk of hospitalization for COPD: results from a Danish longitudinal population study. Eur Respir J. 1997;10:822-27.
18. Canadian Lung Association. Women and COPD: A national report, Ottawa, Canadian Lung Association, 2006.

19. Ernster V, Kaufman N, Nichter M, Samet J, Yoon SY. Women and tobacco: moving from policy to action. Bull World Health Organ. 2000;78(7):891-901.

20. Sarna L, Aguinaga Bialous S. Why tobacco is a women's health issue. Nurs Clin North Am. 2004;39(1):165-80.

21. Siegfried JM. Women and lung cancer: does oestrogen play a role? Lancet Oncol. 2001;2(8):506-513.

22. Camp PG, Goring SM. Gender and the diagnosis, management, and surveillance of chronic obstructive pulmonary disease. Proc Am Thorac Soc. 2007;4:686-91.

23. French CT, Fletcher KE, Irwin RS. Gender differences in health-related quality of life in patients complaining of chronic cough. Chest. 2004;125:482-88.

24. Chapman KR, Tashkin DP, Pye DJ. Gender bias in the diagnosis of COPD. Chest. 2001;119:1691-695.

25. Sinclair A, Tolsma D. Gender differences in asthma experience and disease care in a managed care organization. J Asthma. 2006;40(8):945-53.

26. Belloch A, Perpiñá M, Martínez-Moragón E, de Diego A, Martínez-Francés M. Gender differences in health-related quality of life among patients with asthma. J Asthma. 2003;40(8):945-53.

27. Woods S, Sorscher J, King J, Hasselfeld K. Young adults admitted for asthma: does gender influence outcomes? J Womens Health. 2003;12(5):481-85.

28. Johnson JL, Campbell AC, Bowers M, Nichol A-M. Understanding the social consequences of chronic obstructive pulmonary disease: the effects of stigma and gender. Proc Am Thorac Soc. 2007;4:680-82. 
29. Sarna L, Brown JK, Cooley ME, et al. Quality of life and meaning of illness of women with lung cancer. Oncol Nurs Forum. 2005;32(1):E9-19.

30. Scharf DS. Are there gender differences in smoking cessation, with and without bupropion? Pooled- and meta-analyses of clinical trials of Bupropion SR. Addiction. 2004;99:1462-469.

31. Bohadana A, Nilsson F, Rasmussen T, Martinet Y. Gender differences in quit rates following smoking cessation with combination nicotine therapy: influence of baseline smoking behavior. Nicotine Tob Res. 2003;5(1):111-16.

32. Bjornson W, Rand C, Connett JE, et al. Gender differences in smoking cessation after 3 years in the lung health study. Am J Public Health. 1995;85:223-30.

33. Westmaas JL, Langsam K. Unaided smoking cessation and predictors of failure to quit in a community sample: effects of gender. Addict Behav. 2005;30(7):1405-424.

34. Ward KD, Klesges RC, Zbikowski SM, Bliss RE, Garvey AJ. Gender differences in the outcome of an unaided smoking cessation attempt. Addict Behav. 1997;22(4):521-33.

35. Wetter D, Kenford S, Smith S, Fiore M, Jorenby D, Baker T. Gender differences in smoking cessation. J Consult Clin Psychol. 1999;67(4):555-62.

36. Osler M, Prescott E, Godtfredsen N, Hein H, Schnohr P. Gender and determinants of smoking cessation: a longitudinal study. Prev Med. 2000;29(1):57-62.

37. Killen J, Fortmann S, Varady A, Kraemer H. Do men outperform women in smoking cessation trials? Maybe, but not by much. Exp Clin Psychopharmacol. 2002;10(3):295-301.

38. Etter J-F, Prokhorov A, Perneger TV. Gender differences in the psychological determinants of cigarette smoking. Addiction. 2002;97(6):733-43.
39. Walsh PM, Carrillo P, Flores G, Masuet C, Morchon S, Ramon JM. Effects of partner smoking status and gender on long term abstinence rates of patients receiving smoking cessation treatment. Addict Behav. 2007;32(1):128-36.

40. Pederson AP, Hoyak KAK, Mills S, Camp PG. Reflecting the changing face of chronic obstructive pulmonary disease: sex and gender in public education materials on COPD. Proc Am Thorac Soc. 2007;4:683-85. 